\title{
Micafungin Compared to Fluconazol for Prophylaxis in High Risk Live Donor Liver Transplants: A Randomized Controlled Trial
}

\author{
Yassen $\mathrm{K}^{3}$, Fekry M,, ${ }^{1}$ Kamel $\mathrm{Y}^{3}{ }^{3}$ Mohamed E, ${ }^{2}$ Abdel Aziz A. ${ }^{2}$ \\ Public Health and Community Medicine, ${ }^{1}$ Microbiology, ${ }^{2}$ Anaesthesia and Intensive Care ${ }^{3}$ Departments \\ of Liver Institute, Menoufiya University, Egypt
}

Background and aim of work: High risk liver transplanted recipients are prone to a higher incidence of fungal infections. Aim is to evaluate whether the prophylactic administration of Micafungin compared to standard regime of prophylactic Fluconazol would help reduce fungal infections (Site (urine, wound, throat, drains) and blood invasive), and to monitor their effect on liver graft and renal functions as well as the suggested antifungal therapy duration.

Methods: After local ethics committee approval and consent (11/2012) Recipients between 2012 and 2016 with risk factors as high MELD score, high blood products consumption and evident bile leak on day 1 were randomized for 5 postoperative days (POD) to receive either intravenous prophylactic Micafungin 50 $\mathrm{mg}$ or Fluconazole, $100 \mathrm{mg}$ daily.

Results: 66 adult recipients were included as high risk from 103 transplanted during the study period (33 Micafungin vs 33 Fluconazol). 74 males and 13 females mainly hepatitis $C$. Both Micafungin and fluconazole were comparable for postoperative site fungal infection (Candidia Albicans) (10/43 versus 14/44, $p>0.05$ ) 3 blood isolated invasive fungal infection (IFI) (Candida Albicans) were diagnosed by POD 15, 21 and 25 after discontinuation of Micafungin. Two of the $3 \mathrm{IFI}$ did not respond to an increase of Micafungin dose from $50 \mathrm{mg}$ to a therapeutic dose of $100 \mathrm{mg}$ and died, while one survived. No reported IFI after fluconazole discontinuation were observed. Postoperative urea, creatinine and liver enzymes blood levels were significantly less with micafungin when compared to fluconazole $(P<0.05)$, particularly creatinine clearance on day $5(P=0.001)$.

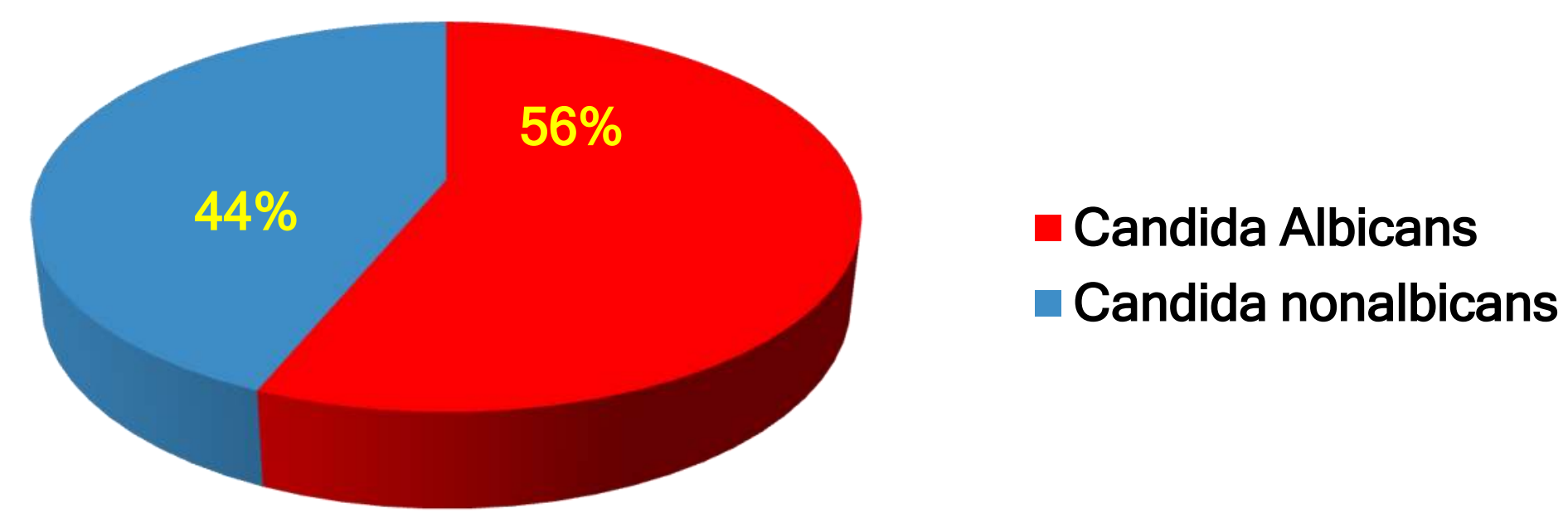

Conclusion: Micafungin has a reduced effect on renal and hepatic graft functions when compared to Fluconazol. The termination of prophylactic Micafungin therapy after discharge from Intensive care lead to the appearance of positive cases of IFI with high mortality. Continuation of prophylactic anti-fungal therapy to beyond 5 days could be necessary to avoid hospital acquired fungal infection in this high risk recipients until final discharge, but the appropriate duration of prophylaxis, resistance and economic impact of using expensive echinocandins need to be studied further. 